\title{
Impact Analysis of Different Types of Online Games Webcast on the Originality of Game Graphics
}

\author{
Niu Tian*, Yang Dong, Zhou Wei \\ School of Law, Southwest Minzu University, Chengdu 610041 \\ *Corresponding Author email: 18215562691@163.com
}

Keywords: Online game; webcast; game graphics; originality.

\begin{abstract}
The development of webcast games has not only brought the heat to the online game webcast industry, but also triggered many legal issues closely related to intellectual property rights. Whether the game pictures are allowed to be regarded as the game work has become one of the major hot topics. This paper attempts to discuss the impact analysis of different game types on the originality of game graphics with the consideration of the characteristics of different types of online games and the combination with some typical cases.
\end{abstract}

\section{Introduction}

The industries of online games and webcast in China have been highly active with an obvious development in recent years. However, the rapid progression has brought many legal issues, for instance, whether the game graphics [1] are allowed to be regarded as the game work has become one of the hot topics of intellectual property rights.

\section{Overview of the Development of Online Game Webcast Industry}

\subsection{The Development Status of the Online Game Webcast Industry}

Online Game means a video game that requires networking to run on the PC-side. It is explained in Baidu Encyclopedia as "a sustainable individual multiplayer online game using the Internet as the transmission medium, the game operator server and the user computer as the processing terminal, and the game client software as the information interaction window for the realization of entertainment, leisure, communication and virtual achievement"[2].

Online game webcast, based on online games, refers to synchronized spreading the process of game players playing different online games to the public through the Internet, television and other media, so that the audience can understand the gamer's strategy and progress of the game in real time [3]. Since its inception in 2013, the scale of the online game webcast industry has increasingly grown, as well as the number of webcast platforms and their users. According to relevant reports, the overall size of China's game live streaming market in 2017 was as high as 8.7 billion yuan, and the number of webcast platforms users reached 220 million. [4] On May 11, 2018, Huya Tv was the first Chinese webcast platform which was officially listed on the New York Stock Exchange.

\subsection{Game Types on Webcast Platforms}

At present, the common domestic webcast platforms mainly include Douyu TV, Panda TV, Huomao TV, Huya TV, Quanmin live, etc., major webcast online games include MOBA, STG and MMORPG. The MOBA games can be subdivided into MOBA-like and MOBA games, while STG (shooter games) can be subdivided into FPS (First-person Shooter) and TPS (Third Person Shooting). It is enumerated that the mainly played games in the following Chinese platforms are shown in Figure 1 


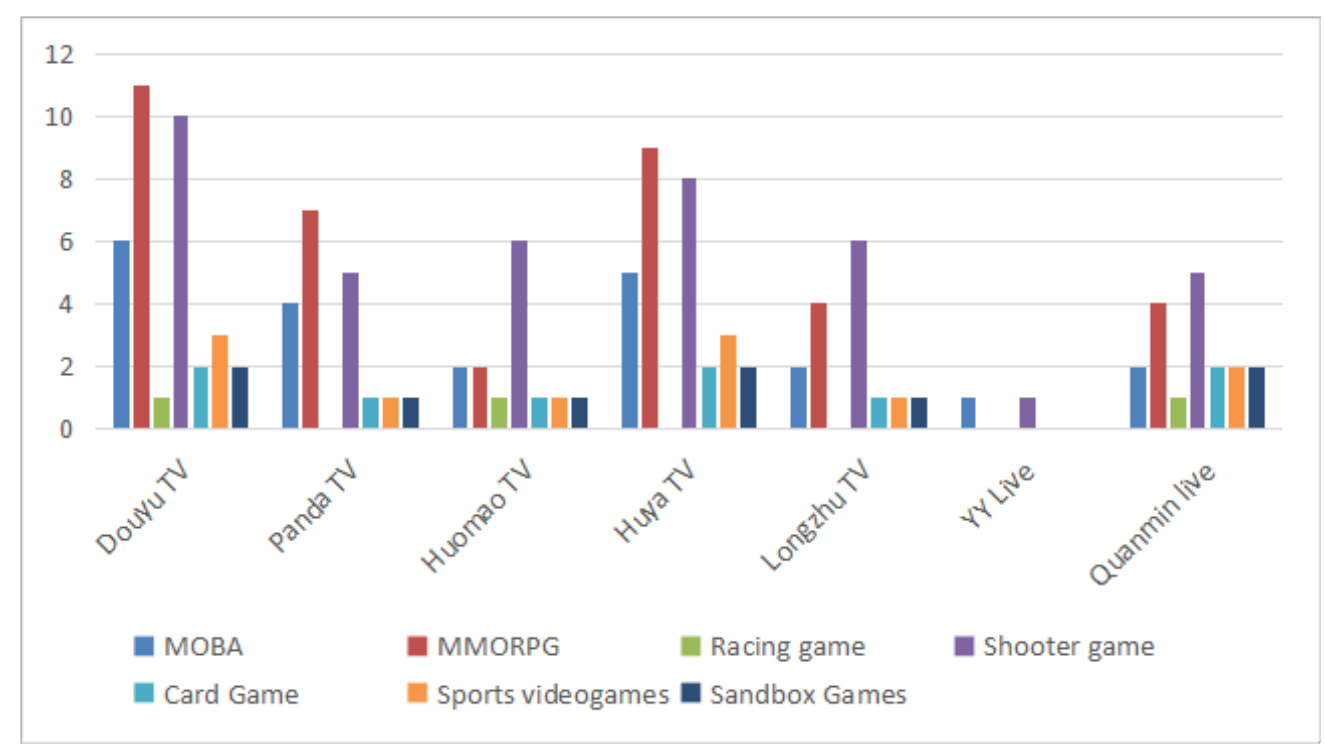

Fig.1 Types and numbers of mainly online game on Chinese webcast platforms [5]

As the Fig.1 shows, the current live streaming games on Chinese webcast platforms include MOBA, MOBA-like, racing, card, MMORPG, shooter, sports and sandbox games. Among them, the most common games are MOBA and MOBA, MMORPG and shooting games, and big E-sports competitions are usually concentrated in these three types of games, which have high competitiveness and strong confrontation, but the difference among the three types of games from the player's operating experience and the game mode is still very tremendous.

\section{Feature Analysis of Different Webcast Games}

\subsection{MOBA}

MOBA is short for Multiplayer Online Battle Arena, translated in Chinese as"Multiplayer online tactical competitive games". It is considered a subclass of Real Time Strategy Games (RTS) in Action real-time strategy (Action RTS, ARTS).

The common MOBA games now on webcast platforms mainly include the following categories, as shown in Table 2-1, in which League of Legends and DOTA2 are both available webcast on all major platforms. This type of game is mainly distinguished from other characters by the external representation of a single character. Each game is divided into two camps to fight against each other's tower defense. Each team has four modes as 1, 3, 5, and 10. Each player can only control one character in the game, and the length of a game usually ranges from 20 minutes to 1 hour.

Table 1 Situation about main MOBA and MOBA-like games on Chinese webcast platforms [7](Note: "0" in Table 2-1 stands for "No", " 1 " stands for "Yes")

\begin{tabular}{|c|c|c|c|c|c|c|}
\hline $\begin{array}{l}\text { Live-broadcasting } \\
\text { platform }\end{array}$ & LOL[6] & DOTA2 & DOTA & $\begin{array}{l}\text { Heroe } \\
\text { s of } \\
\text { the } \\
\text { Storm }\end{array}$ & heroes & $\begin{array}{l}\text { Dream three } \\
\text { kingdoms }\end{array}$ \\
\hline Douyu TV & 1 & 1 & 1 & 1 & 1 & 1 \\
\hline Panda TV & 1 & 1 & 1 & 1 & 0 & 0 \\
\hline Huomao TV & 1 & 1 & 0 & 0 & 0 & 1 \\
\hline Huya TV & 1 & 1 & 1 & 1 & 1 & 1 \\
\hline Longzhu TV & 1 & 1 & 0 & 0 & 0 & 1 \\
\hline YY Live & 1 & 0 & 0 & 0 & 0 & 0 \\
\hline Quanmin live & 1 & 1 & 1 & 0 & 0 & 1 \\
\hline
\end{tabular}


The game graphics of webcast MOBA game is a continuous picture formed by the game streamer through real-time operation, releasing various skills and walking positions in the game. Since different operations at different timings will display different effects, the streamer mainly shows his/her game operation skills and techniques through "virtuoso".

\subsection{MMORPG}

MMORPG is a derivative of the RPG game, which stand for Massive Multiplayer Online Role Playing Game. The online game "King of Kings" introduced by Huacai Software in early 1999 is the first MMORPG introduced to China. [8]The biggest feature of this type of game is role-playing. The game itself has a rich and complete storyline and cultural background, a perfect player character growth system, and strong social interaction. Currently, the most common MMPORG in China are Fantasy Westward Journey, Legend of Mir, Jian Wang 3, World of Warcraft and the latest released Justice Online Treacherous Waters. [9]

The game graphics of MMORPG is how game streamer upgrades, completes the main quest and interacts with others when playing the game.

\subsection{Shooter Game}

Shooter game is the most popular online game category, which can be subdivided into FPS (First-person Shooter), TPS (Third Person Shooting), etc... The first person game is operated from the subjective perspective of the player, while the player in the third person game is like a bystander or controller. Specific online games include CrossFire, Player unknown’s Battlegrounds, Fortnite, Overwatch, and so on.

This kind of game shares great similarity with MOBA games. It is mainly played by players using various weapons to compete against other players, and strives to win in each game. Due to different maps, it can be divided into closed map shooting and sandbox type map design. The latter has a larger tactical space, and the streamer mainly broadcasts a picture of its real-time operation in the game.

\subsection{Card Game}

Card game can be divided into trading and non-trading categories, mainly centered on TCG (Trading card game). It is generally for players to purchase random cards in the game, collect cards, flexibly combine them, and ultimately use strategies to win. Because two sides have different card sets, and random orders to draw cards, the whole game process is ever-changing. For example, the hottest card game "Hearth Stone" uses the card to consume crystal or hero skills to cause damage, and the card-drawing of both sides is randomly generated. The live picture of such games is usually presented to the audience by the streamer in dealing with different types of processing strategies and techniques.

\section{Originality Identification of Game Graphics in Different Types of Webcast Games at Judicial Case}

\section{1 “Shanghai Yaoyu Culture Media Co., Ltd. v. Guangzhou Douyu Network Technology Co., Ltd. Copyright Infringement and Unfair Competition Disputes”[10]}

In February 2015, Shanghai Yaoyu Culture Media Co., Ltd. sued Douyu Tv, the first webcast platform in China, to the court, arguing that Guangzhou Douyu Network Technology Co., Ltd. infringed copyright and constituted unfair competition disputes. Shanghai Yaoyu Company has the authority of the Perfect World Company of Dota2 agent in mainland China to host the competition and obtain the exclusive video broadcasting rights of the event. However, the Guangzhou Douyu Company pirated the event in real time without authorization during the whole course. Due to the particularity and influence of the case, "Yaoyu v. Douyu" was also referred to as "the first case of webcast games in China" by the media.

The Dota2 involved in the case belongs to the MOBA game, and the judge rejected the originality 
of the live game graphics, because the works emphasized by China's copyright law are intellectual achievements that are original in the fields of literature, art and science and can be replicated in some tangible form. However, the Dota2 game event has no prior design of the script, and the screen is only formed by the game players (the streamer) through their respective operations following the game rules. Whether the game graphics of the webcast game event can be regarded as a game work depends on the composition elements such as the commentary content, the captured picture, and the originality of the combination. In fact, the judge in this case has already noticed that the originality of the dynamic picture should be derived from the creative space that the game gives to the streamer (player).

\subsection{Netease Inc. v. Huado Company's "Fantasy Westward Journey Online Game Webcast"[11]}

In 2014, Netease, the author of Fantasy Westward Journey, filed suit against Huaduo Company in the Guangzhou Intellectual Property Court. NetEase clearly pointed out in the lawsuit that live broadcasting, recording and rebroadcasting of Fantasy Westward Journey by Huado Company (Huya $\mathrm{Tv}$ ) constituted infringement of copyright and unfair competition. After the hearing, the Guangzhou Intellectual Property Court affirmed the originality of the game graphics of Fantasy Westward Journey. It is believed that if the game graphics of a game is to be included in the scope of the work, it must be proved that the game graphics has two basic qualities of the work_— "ingenuity" and "reproducibility."

As a typical MMORPG game, Fantasy Westward Journey was considered by the Guangzhou Intellectual Property Court that the game's pictures had a rich storyline, guided by the literary work "Journey to the West", creating distinctive character images and styles, which expressed the unique ideological personality of the creator. Moreover, when inspecting the production process, the game planners and art designers also designed the overall material of the game and encoded the whole game. After the broadcast of the continuous pictures, it is perceived by the viewers of the webpage, thus infringing on the copyright of Netease's presentation of its video game as a movie-like work. Therefore, the distribution of Fantasy Westward Journey game content by Huodu Company (Huya Tv) by means of live broadcasting, recording, and rebroadcasting does infringe the originality of the original work, rather than creating new works.

\section{3 “Douyu Tv v Qiuri, Quanmin live”[12]}

"Qiuri", formerly known as Zhu Hao, was the exclusive signing streamer of "Heart stone" at Douyu Tv. He signed a five-year "Game Interpretation Cooperation Agreement" with Yuqu Company, but later he moved to Quanmin live. Therefore, Yuqu Company requested the court to judge the Xuanmo and Maimiao company infringing on its copyright and causing unfair competition by playing Zhu Hao's "Heart stone" webcast works, which had the copyright of the Yuqu company on Quanmin live.

Whether the dynamic pictures formed by the game operation in this case have copyrightability depends on by the judgement that whether the dynamic pictures belong to a new work different from the online game work itself. If it is only a preset that shows the game work itself, then the picture has no copyrightability. Moreover, the consideration of originality in the dynamic picture should also be combined with the game type and the creative space reserved in the game operation. Different game types have different creative spaces, so the possibilities of forming a work are different. Eventually, the court comprehensively analyzed the skills, strategies and choices of the players, believing that the operation of Hearth Stone is only a demonstration of the game strategy and skill, and it is not original.

\section{Originality Application of Different Types of Webcast Game Graphics}

\subsection{The Concept and Standards of Originality in Works}

It can be seen from the above that if the online live game graphics wants to form a work and is protected by copyright, it is essential to have originality. However, there is a big controversy about 
how this concept should be applied. The European Union's identification of originality is an independent intellectual creation. The relevant cases in the UK reflect that the British copyright law's judgment on originality differs according to the type of work, which covers the author's labor and skills, while France believes that it should reflect the author's personality.

There is no specific provision about this in China's current legislation. Theorists consider originality as ingenuity, which means the author invests certain intellectual labor in the process of creating works, and the works itself has the minimal creativity. [13] Beijing High Court recently issued the "Guidelines for the Trial of Copyright Infringement Cases", which pointed out that the factors of originality are mainly: 1. whether the author completes the creation independently; 2.whether the arrangement of expression reflects the author's choice and judgment. Related scholars conduct an empirical analysis of the current judicial cases, and believe that the criteria for judging originality in practice are: 1 .whether it is a personalized expression; 2 . whether it is the result of choice, trade-off, and processing under the sense of autonomy; 3 . whether it has novelty; 4. whether it will have an impact on the public domain.[14]In addition, in the judicial practice, the originality analysis of specific works is mainly through the comparative analysis of their original elements, that is, whether there are original factors, or whether the same elements have different original requirements. In general, owing to the lack of unified standards, the determination of originality is both strict and loose, mainly depending on the personal opinion of the judicator. Nonetheless, the basic principles of originality determination are unchangeable, and the originality of the work still needs to proceed from the overall, social standards and balance the interests of all parties.

\subsection{Originality Application of Different Types of Webcast game graphics}

The online game webcast pictures refer to ever-changing continuous pictures formed by the computer executing a coded instruction sequence to call the game content according to the player's operation or the autonomous operation of the game program. The originality distinction shows that "the originality threshold of different works is varied"[15]. The game type and the creative space reserved in the game operation are important considerations for weighing whether the game is original or not. Only by analyzing on different types of bases can we recognize the originality application of different types of webcast game graphics.

The game works include two major parts: game programs and game resources. According to the latest "Guidelines for Infringement of Copyright Cases" issued by the Beijing High Court, the resources components of online game such as clothing and props can be composed separately. The static pictures, dynamic pictures and online games itself should be distinguished as different works and receive protection. The online game webcast discussed in this paper mainly involves the retrieval and utilization of game resources, and the diversified game graphics under the operation of the streamer due to different types.

Proceeding from the game types, if the webcast game belongs to an online game that is preset to the player's creative space, when the streamer operates computer to perform codified calling of the original content of the game, the result is not a program preset, but a brand new one. This is the clearest performance in sandbox games, whose core gameplay is creativity, allowing the streamer to play to the fullest, and leaving a great influence on the originality of the game graphics. Such as "Minecraft" [16], a typical sandbox game, in which players (streamers) are free to explore, build, and interact in the game. Creating animals, houses, and cities depends on their own choices.

But the MOBA games mentioned above are characterized by competitiveness and strategy-base. The live game graphics presented by this kind of game usually are the pictures of streamers using different strategies and techniques, elastic blocking to release skills, help the team win instead of creating a new building. Although the live game graphics of this game is continuous, the picture presented is only the original program, and does not form a new expression, nor having originality due to the characteristics of the game type. The same goes for card game. Although the card game is difficult to predict, those cards always exist in the system, while the choices and strategies of each streamer are inconsistent.

However, MMORPG games are different from all the above games. The key to such game is the 
complete storyline and role-playing. It also relies on the plot and upgrade system for the player's viscosity. When the streamer webcasts it on the computer, the continuous picture presented not only covers the resources in the original game library and follows the storyline, but also shows the specific game graphics under the multiplayer participation. Moreover, as MMORPG games keep updated, the third-generation MMORPG gives players more creative space, and players can create own content and gameplay. The continuous picture of such games presented by the streamer is the result of multi-user participation interaction, with creative space and originality.

\section{Conclusion}

In summary, the influence of different types of webcast games on the originality of the game graphics is based on the creative space given to the players by the game, and the two are positively correlated. Whether from the majority of scholars or the newly issued trial guide, they all point out that the game's running picture is original and can form a work. However, they ignore the impact of the game type on the game graphics. Whether the continuous dynamic picture formed by the game graphics under the player or streamer's explanation has originality has to be analyzed on the base of game types. Therefore, it is impossible to determine whether the game graphics forms a work without considering the webcast game types and the specific behaviors of the streamer or player, and exploring the originality of the game graphics.

\section{Acknowledgement}

This paper is funded by the Southwest Minzu University's 2018 Central University Basic Research Business Expenses Special Funds Outstanding Student Training Project: "Impact Analysis of Different Types of Online Games Webcast on the Originality of Game Screens” (2018YXXS14).

\section{References}

[1] Li Mingde, Xu Chao. Copyright Law [M], Beijing: Law Press, 2009.

[2] The Translation Group of the Copyright Law of the Twelve Countries. The Copyright Law of the Twelve Countries [M], Beijing: Tsinghua University Press, 2011.

[3] Guo Rengui, Zhou Hang. Qualitative and Speculative Analysis of Online Game Picture in the Perspective of Copyright [J].Journal of China University of Petroleum (Social Science Edition), 2018, 34(02):66-72.

[4] Wan Qi. On the originality of British copyright law [J]. Intellectual Property, 2017 (11): 94-100.

[5] Li Ming.The Problem of Copyright in Online Game Webcast_-Taking the Qualitative and Ownership of Online game graphics[J].Learning and Practice,2017(06):37-44.

[6] Tang Xianbo, Huang Mingjian, Li Ping. An Empirical Study on the Identification of Originality in Chinese Works[J].Journal of Guizhou Normal University(Social Science Edition),2017(03):151-160.

[7] Li Yang. The Copyright Issue in Online Game Webcast [J]. Intellectual Property, 2017 (01): 14-24.

[8] Wang Qian. Research on the Copyright of Live Video Games [J]. Electronic Property Rights, 2016(02): 11-18.

[9] Wang Qian. On the Copyright Protection of Live Scenes of Sports Events_—Comment on the "Phoenix Network Event Broadcasting Case"[J].Legal Science (Journal of Northwest University of Political Science and Law), 2016,34(01):182-191.

[10] Wang Kun.On the Originality of the Works_—based on the Scientific Construction of the Concept of Works [J].Intellectual Property, 2014(04):15-22. 